\title{
Recurrence Risk for Autism Spectrum Disorders: A Baby Siblings Research Consortium Study
}

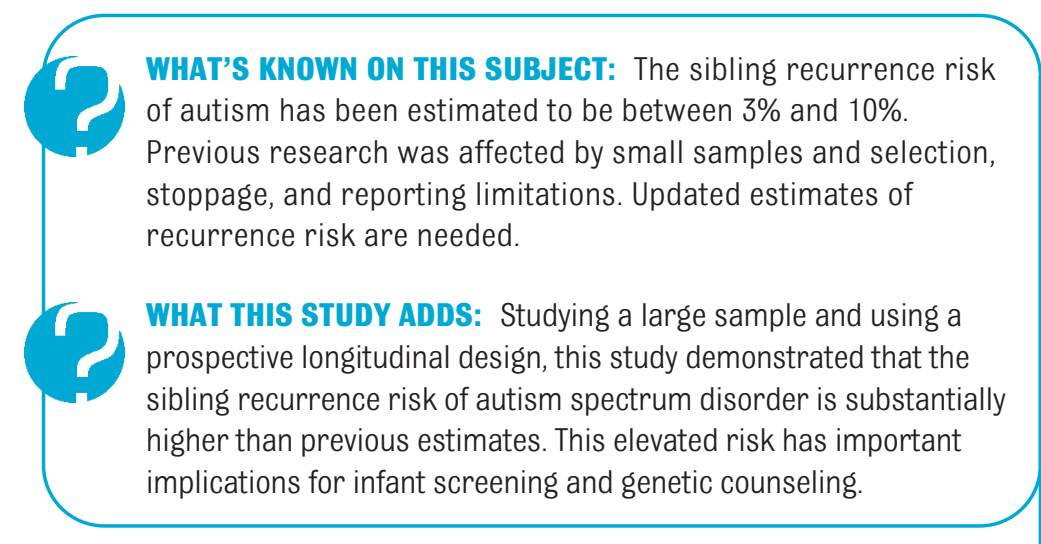

abstract

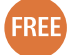

OBJECTIVE: The recurrence risk of autism spectrum disorders (ASD) is estimated to be between $3 \%$ and $10 \%$, but previous research was limited by small sample sizes and biases related to ascertainment, reporting, and stoppage factors. This study used prospective methods to obtain an updated estimate of sibling recurrence risk for ASD.

METHODS: A prospective longitudinal study of infants at risk for ASD was conducted by a multisite international network, the Baby Siblings Research Consortium. Infants ( $n=664)$ with an older biological sibling with ASD were followed from early in life to 36 months, when they were classified as having or not having ASD. An ASD classification required surpassing the cutoff of the Autism Diagnostic Observation Schedule and receiving a clinical diagnosis from an expert clinician.

RESULTS: A total of $18.7 \%$ of the infants developed ASD. Infant gender and the presence of $>1$ older affected sibling were significant predictors of ASD outcome, and there was an almost threefold increase in risk for male subjects and an additional twofold increase in risk if there was $>1$ older affected sibling. The age of the infant at study enrollment, the gender and functioning level of the infant's older sibling, and other demographic factors did not predict ASD outcome.

CONCLUSIONS: The sibling recurrence rate of ASD is higher than suggested by previous estimates. The size of the current sample and prospective nature of data collection minimized many limitations of previous studies of sibling recurrence. Clinical implications, including genetic counseling, are discussed. Pediatrics 2011;128:e488-e495

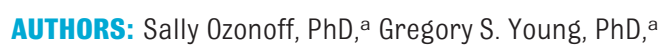
Alice Carter, PhD, ${ }^{b}$ Daniel Messinger, PhD, ${ }^{,}$Nurit Yirmiya, PhD, donnie Zwaigenbaum, MD, e Susan Bryson, PhD, ${ }^{f}$ Leslie J. Carver, PhD, 8 John N. Constantino, MD, ${ }^{\text {h Karen }}$ Dobkins, PhD, 8 Ted Hutman, PhD,' Jana M. Iverson, PhD, j. Rebecca Landa, PhD, ${ }^{\mathrm{k}}$ Sally J. Rogers, PhD, ${ }^{a}$ Marian Sigman, PhD,' and Wendy L. Stone, PhDm

${ }^{a}$ Department of Psychiatry, University of California-Davis, Sacramento, California; ${ }^{b}$ Department of Psychology, University of Massachusetts Boston, Boston, Massachusetts; 'Department of Psychology, University of Miami, Miami, Florida; 'Department of Psychology, Hebrew University of Jerusalem, Jerusalem, Israel; ${ }^{e}$ Department of Pediatrics, University of Alberta, Alberta, Canada; ${ }^{f}$ Department of Pediatrics, Dalhousie University, Halifax, Nova Scotia, Canada; ${ }^{g}$ Department of Psychology, University of California-San Diego, San Diego, California; ${ }^{h}$ Department of Psychology, Washington University, St Louis, Missouri;

'Department of Psychiatry and 'Division of Child and Adolescent Psychiatry Neuropsychiatric Institute and Hospital, University of California-Los Angeles, Los Angeles, California; jDepartment of Psychology, University of Pittsburgh, Pittsburgh, Pennsylvania; ${ }^{k}$ Center for Autism and Related Disorders (CARD), Kennedy Krieger Institute, Baltimore, Maryland; and ${ }^{m}$ Department of Psychology, University of Washington, Seattle, Washington

KEY WORDS

autism, recurrence, sibling risk

\section{ABBREVIATIONS}

ASD—autism spectrum disorders

ADOS-Autism Diagnostic Observation Schedule

$\mathrm{Cl}$-confidence interval

$d f$-degrees of freedom

Drs Ozonoff and Young had full access to all of the data in the study and take responsibility for the integrity of the data and the accuracy of the data analysis. Drs Ozonoff, Young, Carter, Messinger, Yirmiya, Zwaigenbaum, Bryson, Carver, Constantino, Iverson, Rogers, and Sigman contributed to the study concept and design; Drs Ozonoff, Young, Messinger, Zwaigenbaum, Bryson, Carver, Constantino, Dobkins, Hutman, Iverson, Landa, Rogers, and Stone contributed to the acquisition of data; Drs Ozonoff, Young, Carter, Yirmiya, Zwaigenbaum, Bryson, Carver, Constantino, Hutman, and Stone contributed to the analysis and interpretation of data; Drs Ozonoff and Young drafted of the manuscript; Drs Ozonoff, Young, Carter, Messinger, Yirmiya, Zwaigenbaum, Bryson, Carver, Constantino, Dobkins, Hutman, Iverson, Landa, Rogers, Sigman, and Stone contributed to the critical revision of the manuscript for important intellectual content; Drs Young, Ozonoff, and Zwaigenbaum contributed to the statistical analysis; Drs 0zonoff, Young, Messinger, Yirmiya, Zwaigenbaum, Bryson, Carver, Constantino, Dobkins, Iverson, Landa, Rogers, Sigman, and Stone obtained funding; Drs Ozonoff, Young, Messinger, Hutman, and Stone contributed to administrative, technical, or material support; and Drs Ozonoff, Young, Carter, Messinger, Yirmiya, Carver, Rogers, and Sigman contributed to the study supervision.

(Continued on last page) 
Autism spectrum disorders (ASDs) are among the most common neurodevelopmental disorders, with recent surveillance efforts indicating that 1 in 110 American children meet criteria for ASD. ${ }^{1}$ The gender ratio is highly skewed, with $\sim 80 \%$ of affected individuals being male. There is strong evidence that genetic factors play a critical role in vulnerability to $A S D,{ }^{2}$ with heritability estimates from twin studies as high as $90 \%{ }^{3}$ Moreover, there have been recent advances in identifying specific genetic causes of ASD, such as genomic copy-number variants in genes involved in synaptic cell adhesion and related pathways, which have been identified in as many as $7 \%$ to $10 \%$ of people with ASD. ${ }^{4,5}$ However, there still are many individuals with ASD for whom the etiology is not yet known.

An important measure of genetic contribution is the risk of recurrence in siblings. Previous studies ${ }^{6-8}$ have examined the rate of ASD in families who already have 1 affected child, with recurrence estimates ranging from $3 \%$ to $10 \%$. However, only a few studies have taken into account the effects of stoppage ${ }^{9}$ (the tendency for families to halt reproduction after the diagnosis of an affected child) by studying families in which there are later-born siblings. A large epidemiologic survey of autism in Utah in the 1980s reported a recurrence risk of $8.6 \%$ in siblings born after an affected child. ${ }^{10}$ An even higher risk to later-born siblings of $14.2 \%$ recently was reported in a large US registry of children with ASD. ${ }^{11}$ The Utah study found that the risk to laterborn children was approximately twice as high if the first affected child was a female, which is consistent with a multifactorial threshold model of transmission, in which risk is elevated for relatives of a proband in which the condition is less common. 10,12 More recent studies, ${ }^{13,14}$ however, have pro- vided mixed evidence for this threshold model.

Although reproductive stoppage leads to underestimates of sibling recurrence risk, ascertainment biases and overreporting can lead to inflation of sibling recurrence risk. ${ }^{15,16}$ Parents who already have an affected child may focus more attention on the new infant's development, ${ }^{17}$ which may increase the probability of both truepositive and false-positive identification. Samples may be more or less biased by the strictness of the inclusion criteria, the age of participant enrollment, and diagnostic methods. Optimal estimates require both population-based epidemiologic methods to assure that the sample is maximally representative of all families who have a child with ASD and expert direct assessment to supplement parent-report methods. ${ }^{15}$

The present study reports on data from a large cohort of infants collected as part of an international collaboration to study the earliest signs of ASD in infants with an older affected sibling. Infants were followed prospectively through the window of risk for symptom emergence. Diagnostic assessments were performed at 36 months of age by expert examiners. The prospective design, directassessment methods, gold-standard diagnostic procedures, young age at enrollment, geographic diversity of recruitment, and large sample size minimize many methodological limitations of previous research and provide updated estimates of recurrence risk.

\section{PATIENTS AND METHODS}

The Baby Siblings Research Consortium is an international network supported by Autism Speaks that pools data from individually funded research sites to study the development of high-risk infants. The present analyses were conducted on data contrib- uted from 12 sites (University of Alberta, Dalhousie University, Kennedy Krieger Institute, McMaster University, University of California-Davis, University of California-Los Angeles, University of California-San Diego, University of Miami, University of Pittsburgh, University of Toronto, Vanderbilt University, and Washington University St Louis) that had sufficiently similar procedures and common measures to permit data pooling. Institutional review board approval to collect and analyze deidentified data from all sites was obtained.

Infant participants were later-born biological siblings of a child with ASD. Less than $1 \%$ of participants were halfsiblings ( 6 of 664). Exclusion of these participants did not change the results, so they were retained in the analyses. Diverse community recruitment strategies were used. All sites recruited participants from clinics and agencies serving individuals with ASD, community events (lectures, health fairs, and local autism society meetings) targeted at families affected by ASD, and other studies of ASD at their respective universities. Most sites also recruited participants through Web sites targeted to ASD, word of mouth (parents referring other parents), and fliers posted in the community. A few sites also used mailings and media announcements to recruit participants. Inclusion criteria included a documented diagnosis of autistic disorder, Asperger disorder, or pervasive developmental disorder not otherwise specified in the affected sibling (hereafter called the proband) and no identified neurologic or genetic condition in the infant or proband that could account for an ASD diagnosis (eg, fragile $X$ syndrome). All sites verified the clinical diagnosis of the proband. Most sites collected standardized diagnostic assessments (eg, the Autism Diagnostic Observation Schedule [ADOS]) and/or 


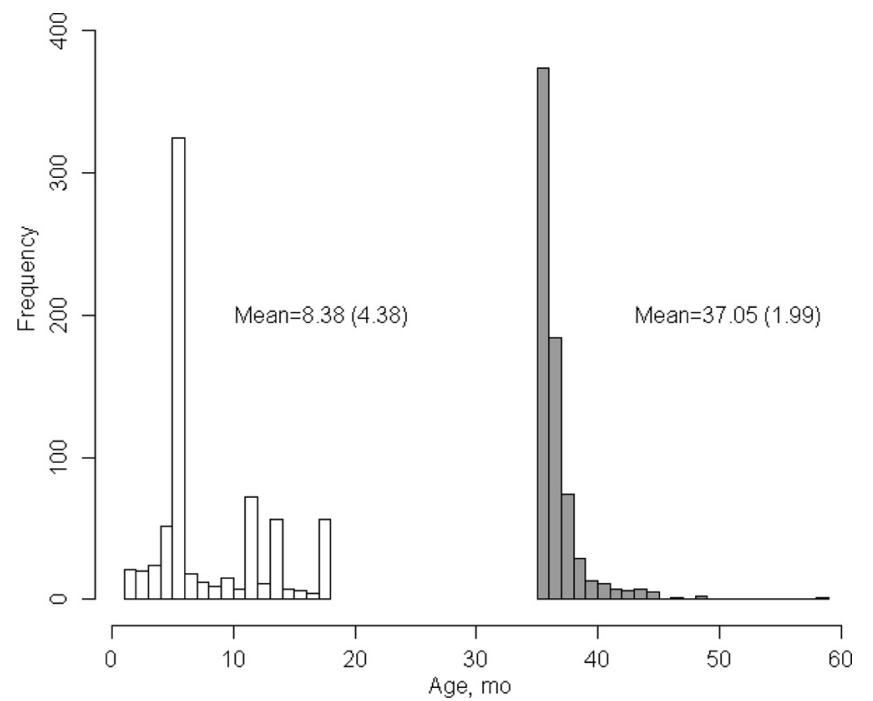

FIGURE 1

Frequencies of age at first visit and age at outcome.

parent diagnostic interviews (eg, the Autism Diagnostic Interview-Revised or the Social Communication Questionnaire) as part of the proband diagnostic verification process.

Additional inclusion criteria were maximum enrollment age of 18 months, minimum outcome assessment age of 36 months (see Fig 1), and the availability of both a clinical diagnosis and an ADOS. ${ }^{18}$ For families with multiple enrolled infants, only 1 participant per family, the infant recruited at the youngest age, was included. This resulted in a total sample size of 664 participants.

The ADOS and the Mullen Scales of Early Learning were administered at 36 months of age. The ADOS is a standardized protocol that measures symptoms of ASD and yields an empirically derived cutoff for ASD. ${ }^{18}$ Psychometric studies report high interrater reliability and agreement in the diagnostic classification for individuals aged 24 months and older. ${ }^{18}$ The MulIen Scales of Early Learning is a standardized developmental test for children from birth to age 68 months that measures nonverbal cognitive, language, and motor skills. ${ }^{19}$ The Mullen subscales have excellent internal consistency and test-retest reliability. Demographic information also was collected at each site. The race and ethnicity of the infant were reported by parents using categories specified by the National Institutes of Health, which were then collapsed for analysis into a dichotomous variable (non-Hispanic white versus other race/ethnicity). Maternal and paternal education were measured on a 4-point scale indicating high school, some college, college degree, or graduate degree. Maternal and paternal ages at the birth of the child were measured as continuous variables. Birth order of the infant was recorded as a 3-level variable (second born, third born, and fourth born or later). A dichotomous variable was created indicating whether the infant's family was simplex (1 older sibling with ASD $[n=582]$ ) or multiplex (more than 1 older sibling with ASD [ $n=37]$ ). On the basis of the 36-month assessment, participants were classified into 1 of 2 outcome groups. The ASD outcome group included participants who scored above the ASD cutoff of the ADOS and received a clinical diagnosis of autistic disorder or pervasive devel- opmental disorder not otherwise specified according to an expert clinician at each site. The non-ASD outcome group included all other participants.

For the statistical approach, hierarchical generalized linear modeling was used to model outcome as a binomial distribution using a logit-link function. Although preliminary analyses revealed site heterogeneity in recurrence risk, site did not interact with or moderate the effect of any variables in predicting prevalence estimates of ASD outcome. Therefore, only the main effect of site was included as a random effect to account for site heterogeneity in all models. Potential associations of demographic variables (race/ethnicity, parental education, and parental age) with outcome were examined first. Child-specific variables (infant gender, multiplex family status, and proband severity) were examined in subsequent models. Variables that were significant predictors of outcome were retained in the model such that all subsequent analyses controlled for the retained variables. Main and interaction effects were tested by using $\chi^{2}$ tests of differences between goodness-of-fit values ( -2 log-likelihood values) for nested models with and without the effect of interest, using the difference in model parameters as the degrees of freedom. Relative risk and respective confidence intervals (Cls) were estimated using a Poisson quasilikelihood method using SAS Proc Genmod (SAS Institute, Inc, Cary, NC). All other analyses were conducted using $\mathrm{R}$ version 2.9.1 (Vienna Austria), using the Ime4 package for multilevel modeling.

\section{RESULTS}

There were 132 infants (29 female) who met criteria for ASD at outcome, yielding an estimated recurrence rate of $18.7 \%$ (95\% Cl: 13.34-25.5). Of these 132 participants, 54 (40.9\%) received a 
TABLE 1 Descriptive Characteristics of the Sample

\begin{tabular}{lcr}
\hline & Descriptor & $N$ \\
\hline Mean age at enrollment, mo (SD) & $8.4(4.4)$ & 664 \\
Gender, \% male & 55.6 & 663 \\
Race/ethnicity, \% other & 16.0 & 657 \\
Birth order, \% third-born or later & 39.7 & 458 \\
Gender of proband, \% male & 84.2 & 658 \\
Multiplex status, \% with >1 affected older sibling & 6.0 & 619 \\
Maternal education, \% with college degree or higher & 77.1 & 365 \\
Paternal education, \% with college degree or higher & 74.3 & 338 \\
Mean maternal age, y (SD) & $34.5(4.4)$ & 566 \\
Mean paternal age, y (SD) & $36.9(5.2)$ & 563 \\
\hline
\end{tabular}

clinical diagnosis of autistic disorder and 78 (59.1\%) received a clinical diagnosis of pervasive developmental disorder not otherwise specified.

Table 1 includes demographic characteristics of the sample. Analyses of demographic variables as predictors of outcome revealed no significant main effects for race or ethnicity $\left(\chi^{2}=2.6\right.$, degrees of freedom $[d f]=1, P=.10)$, maternal education $\left(\chi^{2}=2.3, d f=3\right.$, $P=.52)$, paternal education $\left(\chi^{2}=4.7\right.$, $d f=3, P=.20)$, or maternal age at birth of the child $\left(\chi^{2}=0.8, d f=1, P=.38\right)$. There was a nonsignificant trend $\left(\chi^{2}=\right.$ 2.9, $d f=1, P=.09$ ) for group differences in paternal age, with fathers of participants with ASD outcomes being slightly younger than fathers of children with non-ASD outcomes. Birth order was not a significant predictor of outcome $\left(\chi^{2}=\right.$ $3.1, d f=2, P=.21$ ), nor was age at study enrollment $\left(\chi^{2}=1.1, d f=1, P=.30\right)$.

The gender of the infant significantly predicted outcome $\left(\chi^{2}=34.9, d f=1\right.$, $P<.001$ ), with $26.2 \%$ (95\% Cl: $19.2-$ 34.6) of male infants receiving an ASD diagnosis versus $9.1 \%$ (95\% Cl: $5.7-14.2$ ) of female infants. The estimated relative risk for gender was 2.8 ( $95 \% \mathrm{Cl}: 1.9-4.0$ ), indicating an almost threefold increase in the risk of an ASD outcome in male relative to female siblings.

Analysis of an ASD diagnosis at outcome as a function of family multiplex status revealed a significant main effect above and beyond infant gender $\left(\chi^{2}=8.0, d f=1, P<.01\right)$. The esti- mated relative risk of $2.2(95 \% \mathrm{Cl}: 1.4-$ 3.3) indicated a twofold increase in the probability of an ASD diagnosis at outcome for infant siblings who had multiple older affected siblings $(32.2 \%$ [95\% Cl: $21.8-44.7)$ relative to those who had only 1 older affected sibling (13.5\% [95\% Cl: 8.4-20.9). In supplementary analyses, simplex families also were defined more stringently as those who have at least 1 unaffected older sibling, in addition to the affected proband and the infant. When analysis was restricted to only families with 3 or more children (total: $n=380$, simplex: $n=343$, multiplex: $n=37$ ), the recurrence rate of $32.2 \%$ in multiplex families continued to be significantly
FIGURE 2 higher than the simplex rate of $20.1 \%$ $\left(\chi^{2}=9.54, d f=1, P=.002\right)$. There was no interaction between multiplex status and gender $(P=.28)$. Figure 2 displays the estimated proportions of ASD outcomes as a function of infant gender and family multiplex status.

The gender of the older sibling was considered next, building on the previous model, which included infant gender and multiplex status as predictors. There was no main effect of proband gender in predicting an ASD outcome $(P=.20)$, and no two-way or threeway interactions between proband gender and infant gender or multiplex status (all $P$ values $>.50$ ). The lack of effect for proband gender persisted even when considering models without infant gender or multiplex status $(P=.52)$.

Finally, we examined proband functioning levels, as measured by fullscale IQ and ADOS scores, as predictors of ASD outcome, above and beyond infant gender and multiplex status. Full-scale IQ was available for 210 probands (30.6\%), using a number of different IQ tests. All scores were

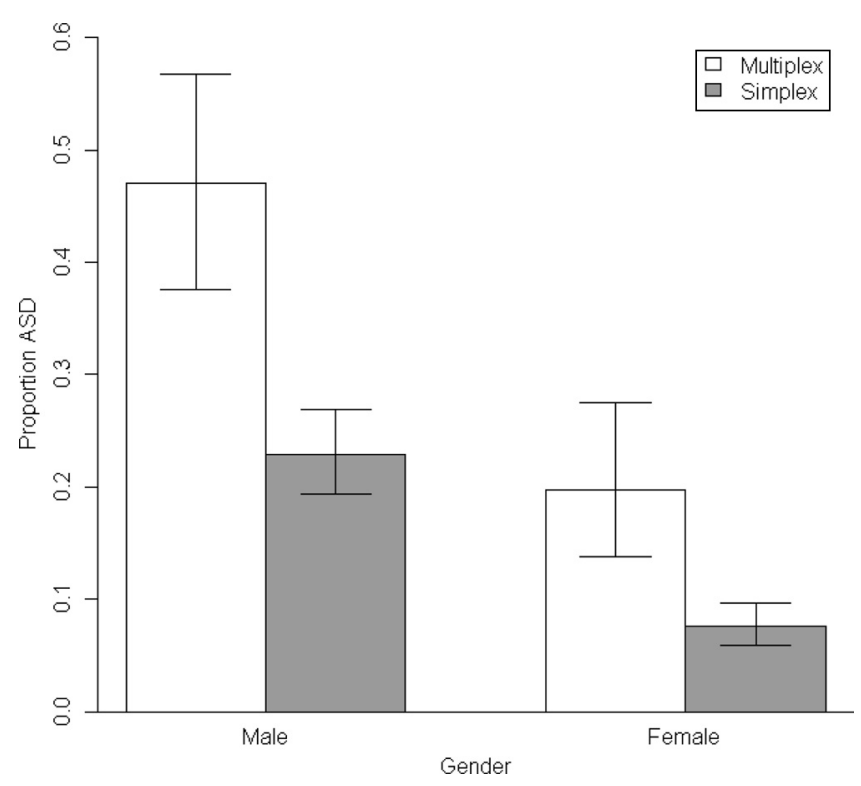

Proportion of ASD outcome according to infant gender and family multiplex status. 
converted to standard scores, with a mean of 100 and an SD of 15. Results revealed no significant effect of proband intellectual functioning on ASD outcome, above and beyond gender and multiplex status $\left(\chi^{2}=0.01, d f=1\right.$, $P=$.92; ASD outcome mean $=71.9$, non-ASD outcome mean $=80.7$ ). Removing multiplex status from the model, the effect remained nonsignificant. ADOS scores were available for $54.5 \%$ of the probands $(n=374)$. Proband symptom severity, as measured by the social-communication algorithm score of the ADOS, was also not predictive of outcome, above and beyond gender and multiplex status $\left(\chi^{2}=2.5\right.$, $d f=1, P=.11$; ASD outcome mean $=$ 15.6, non-ASD outcome mean $=14.4$ ). Without multiplex status included in the model, the effect for proband symptom severity remained nonsignificant.

\section{DISCUSSION}

The current study is the largest prospective investigation of ASD sibling recurrence yet conducted. The primary finding was a substantially higher rate of ASD in infant siblings of children with ASD than previously documented. Earlier investigations reported recurrence estimates ranging from $3 \%$ to $14 \%,{ }^{6-8,10-12,14}$ whereas in this study, $18.7 \%$ of infants with at least 1 older sibling with ASD developed the disorder. The 2 strongest predictors of an ASD diagnosis were the gender of the infant and the number of affected older siblings. Male gender and multiplex family status were independent and significant predictors of an ASD outcome, with a 2.8-fold increase in the risk for ASD for male infants $(25.9 \%$ of high-risk male infants versus $9.6 \%$ of high-risk female infants) and an additional twofold increase in risk if there was more than 1 older affected sibling (13.5\% of simplex versus $32.2 \%$ of multiplex). The increased risk for male infants replicates previous research.10,11 The recurrence rate for multiplex families reported here $(32.2 \%)$ is similar to that found in an earlier populationbased study conducted in Utah (35.3\%). ${ }^{10}$ Previous investigations also suggested that the gender of the proband was associated with recurrence rates, ${ }^{10,14}$ with ASD outcomes more likely if the older affected child was female. The current data did not support such a threshold polygenic model of inheritance, in that similar rates of recurrence were found in families with male and female probands, as previously reported by others. ${ }^{13}$ Additional proband, demographic, and family factors, such as proband IQ and autism severity, infant race, ethnicity and birth order, and parental education and age, also did not predict outcome. There was variability across sites in ASD outcome rates, which may reflect geographic diversity, regional variation, and/or method differences. Site heterogeneity was accounted for as a random variable in all statistical models and did not interact with any predictors of outcome.

The design of the current investigation minimized many of the limitations of earlier research, such as stoppage, overreporting, and ascertainment bias. ${ }^{15,16}$ Stoppage, or the tendency of couples with an affected child to stop reproducing, leads to an underestimate of recurrence rate if uncorrected. ${ }^{9}$ Earlier studies of large unrestricted samples reported that between $4 \%$ and $10 \%$ of families had more than 1 affected child, ${ }^{6-8,11}$ whereas studies that restricted the sample to families with later-born siblings reported higher sibling recurrence rates of between $9 \%$ and $14 \% .{ }^{10,11}$ Stoppage was addressed in the current investigation, by design, through studying only families with later-born siblings.

Overreporting is a second threat to the estimation of sibling recurrence risk.
Because of limitations in time and resources, the affected status of children in previous studies often was determined by parent report or record review, ${ }^{11,13}$ which has been demonstrated to inflate recurrence rate estimates..$^{15}$ The present study addressed overreporting biases through prospective data collection and diagnostic methods that combined structured, reliable assessment tools with expert clinical diagnosis. Diagnostic outcome was determined at 36 months, an age at which multiple studies have documented excellent diagnostic stability, with over $85 \%$ of children retaining a diagnosis several years later ${ }^{20,21}$ Because outcome was determined before the age that milder forms of ASD, such as Asperger disorder, are accurately diagnosed, ${ }^{22}$ the true recurrence rate may in fact be higher than that reported here.

There are several types of ascertainment bias that may affect recurrence rate estimates, particularly in samples such as this one, which were not epidemiologically ascertained. Of primary interest for the present study is the overinclusion of families who have developmental concerns about their later-born infant. Overselection of infants with preexisting developmental delays was minimized in the present investigation by the early age at enrollment, with two-thirds of the sample recruited before the age of 6 months, when behavioral signs and parent concerns of ASD are rare. ${ }^{17,23-26}$ That there were no effects of age at enrollment on rates of ASD outcomes suggests that overselection was not a significant bias in the present study.

Comparing the current sample to population-based studies of children with ASD also is relevant to evaluating ascertainment bias. The gender ratios of both the probands and the infants with ASD outcomes in this study were similar to those reported in the general ASD population. ${ }^{27,28}$ If multiplex 
families were overrepresented in the current sample, this could elevate recurrence rates, but this was not the case. The current sample was $6 \%$ multiplex (before the birth of the infant), whereas other studies report multiplex rates of approximately $10 \% .{ }^{10,11,14}$ Together, this information suggests that the recurrence rates provided by this study were not overly biased, despite the fact that the sample was not epidemiologically ascertained. However, the true rate of sibling recurrence in the general population of families affected by ASD will ideally be estimated in the future through large population-based studies.

These results have significant familyplanning and genetic-counseling implications. ${ }^{29,30}$ At the present time, genetic counseling for ASD is constrained by the fact that currently cited risk estimates are largely on the basis of data from the 1980s and 1990s, when earlier, less inclusive versions of the Diagnostic and Statistical Manual of Mental Disorders were in use. The updated information provided in this report will give families risk estimates that more accurately reflect recurrence as defined by current diagnostic practice (ie, Diagnostic and Statistical Manual of Mental Disorders, 4th Edition, Text Revision). Many families actually believe that the risk to later-born siblings is higher than either the current investigation or previous studies suggest it to be. ${ }^{31,32}$ If families base reproductive decisions on perceived risk of recurrence, it is important that they receive updated information about these risks. Genetic counseling most often is provided for mendelian disorders and is a complex undertaking for disorders of multifactorial inheritance that are influenced by multiple unknown susceptibility genes and other factors. Therefore, it is critical that these data are provided to families in a sensitive manner, with extensive counseling that helps them evaluate risk as we understand it at this time. ${ }^{33}$ It is important to convey that recurrence estimates are on the basis of group averages, and, in most cases, it is not yet possible to counsel parents regarding individual levels of risk. A thorough genetic work-up is essential as part of the etiologic investigation for all individuals with ASD and may have important implications for risk counseling. ${ }^{34}$ DNA collection for genotyping the current high-risk sample is underway and may, in the future, yield critical information about genetic etiologies of ASD.

Finally, this study highlights the importance of routine surveillance and rapid referral for infant siblings of children with ASD. Given the higher-thanexpected recurrence rates, particularly for male infants and multiplex families, it is critical that primary care professionals closely monitor the development of infants who have older siblings with ASD, screening them routinely at well-child visits using a tool appropriate for infants. ${ }^{35-38}$ The red flags identified should be followed by immediate referral for infant intervention rather than adopting a "wait-andsee" attitude because early specialized intervention is considered best practice for $\mathrm{ASD}^{39,40}$ and may represent the best hope for reducing symptoms and overall disability in high-risk infants who are developing ASD. ${ }^{41}$

\section{CONCLUSIONS}

The sibling recurrence rate of ASD is substantially higher than suggested by previous estimates. The size of the current sample and the prospective nature of the data collection minimized many limitations of previous studies of sibling recurrence, including ascertainment bias, stoppage, and overreporting. The elevated risk has impor- tant implications for infant screening and genetic counseling.

\section{ACKNOWLEDGMENTS}

This work was supported by the following grants to the principal investigators at each site: National Institutes of Health (NIH) R01 MH068398 (to Drs 0zonoff, Rogers, and Young); NIH R01 DC10290 (to Dr Carter); NIH R01 HD047417 (to Dr Messinger); NIH R01HD057284 (to Drs Messinger and Stone); the United States-Israel Binational Science Foundation 94-66/3 and 97-00073 (to Drs Yirmiya and Sigman); the Canadian Institute for Health Research 62924 and 102665 (to Drs Zwaigenbaum and Bryson); NIH R01 HD042541 (to Dr Constantino); NIH R01 HD052804-01 (to Drs Dobkins and Carver); NIH R01 HD54979 (to Dr Iverson); $\mathrm{NIH} \quad$ U54 MH066417 and NIH R01 MH059630 (to Dr Landa); NIH U54 MH068172 (to Dr Sigman and Hutman); and NIH R01 HD043292 (to Dr Stone). Autism Speaks provided additional funding to Drs Ozonoff and Young for the creation of a multisite database, data analysis, and writing. The study sponsors had no role in the design and conduct of the study; the collection, management, analysis, and interpretation of the data; or the preparation, review, and approval of the manuscript.

We are grateful to Geraldine Dawson, PhD, Autism Speaks Chief Science Officer, and Alycia Halladay, PhD, Autism Speaks Director of Research for Environmental Sciences, for their uncompensated assistance in organizing the Baby Siblings Research Consortium (BSRC). Helen Tager-Flusberg, PhD, Boston University School of Medicine, provided helpful insights (uncompensated) in her role on the BSRC Executive Committee. Compensated editorial support for the preparation of this article was provided by Diane Larzelere (University of California-Davis). 


\section{REFERENCES}

1. Autism and Developmental Disabilities Monitoring Network Surveillance Year 2006 Principal Investigators; Centers for Disease Control and Prevention (CDC). Prevalence of autism spectrum disorders: Autism and Developmental Disabilities Monitoring Network, United States, 2006. MMWR Surveill Summ. 2009; 58(10):1-20

2. Losh M, Sullivan PF, Trembath D, Piven J. Current developments in the genetics of autism: from phenome to genome. J Neuropathol Exp Neurol. 2008;67 (9):829-837

3. Bailey A, Le Couteur A, Gottesman I, et al. Autism as a strongly genetic disorder: evidence from a British twin study. Psychol Med. 1995;25(1):63-77

4. Cook EH Jr, Scherer SW. Copy number variations associated with neuropsychiatric conditions. Nature. 2008;16(7215):919-923

5. Pinto D, Pagnamenta AT, Klei L, et al. Functional impact of global rare copy number variation in autism spectrum disorders. $\mathrm{Na}$ ture. 2010;466(7304):368-372

6. Chakrabarti S, Fombonne E. Pervasive developmental disorders in preschool children. JAMA. 2001;285(24):3093-3099

7. Icasiano F, Hewson P, Machet P, Cooper C, Marshall A. Childhood autism spectrum disorder in the Barwon region: a community based study. J Paediatr Child Health. 2004; 40(12):696-701

8. Lauritsen MB, Pedersen CB, Mortensen PB. Effects of familial risk factors and place of birth on the risk of autism: a nationwide register-based study. J Child Psychol Psychiatry. 2005;46(9):963-971

9. Jones M, Szatmari P. Stoppage rules and genetic studies of autism. J Autism Dev Disord. 1988;18(1):31-40

10. Ritvo ER, Jorde LB, Mason-Brothers A, et al. The UCLA-University of Utah Epidemiologic survey of autism: recurrence risk estimates and genetic counseling. Am J Psychiatry. 1989;146(8):1032-1036

11. Constantino JN, Zhang Y, Frazier T, Abbacchi, AM, Law P. Sibling recurrence and the genetic epidemiology of autism. Am J Psychiatry. 2010;167(11):1349-1356

12. Jorde LB, Hasstedt SJ, Ritvo ER, et al. Complex segregation analysis of autism. Am J Hum Genet. 1991;49(5):932-938

13. Goin-Kochel RP, Abacchi A, Constantino JN. Lack of evidence for increased genetic loading for autism among families of affected females: a replication from family history data in two large samples. Autism: Int J Res Prac. 2007;11(3):279-286

14. Sumi S, Taniai H, Miyachi T, Tanemura M.
Sibling risk of pervasive developmental disorder estimated by means of an epidemiologic survey in Nagoya, Japan. J Hum Genet. 2006;51(6):518-522

15. Guo SW. Inflation of sibling recurrence-risk ratio due to ascertainment bias and/or overreporting. Am J Hum Genet. 1998;63(1): 252-258

16. Zhao X, Leotta A, Kustanovich V, et al. A unified genetic theory for sporadic and inherited autism. Proc Natl Acad Sci USA. 2007; 104(31):12831-12836

17. Ozonoff S, Young GS, Steinfeld MB, et al. How early do parent concerns predict later autism diagnosis? J Dev Behav Pediatr. 2009; 30(5):367-375

18. Lord C, Risi S, Lambrecht L, et al. The autism diagnostic observation schedule-generic: a standard measure of social and communication deficits associated with the spectrum of autism. J Autism Dev Disord. 2000; 30(3):205-223

19. Mullen EM. Mullen Scales of Early Learning. Circle Pines, MN: American Guidance Service; 1995

20. Charman T, Taylor E, Drew A, et al. Outcome at 7 years of children diagnosed with autism at age 2: predictive validity of assessments conducted at 2 and 3 years of age and pattern of symptom change over time. $J$ Child Psychol Psychiatry. 2005;46(5): 500-513

21. Turner LM, Stone WL. Variability in outcome for children with an ASD diagnosis at age 2. $J$ Child Psychol Psychiatry. 2007;48(8): 793-802

22. McConachie H, Le Couteur A, Honey E. Can a diagnosis of Asperger syndrome be made in very young children with suspected autism spectrum disorder? J Autism Dev Disord. 2005;35(2):167-176

23. Landa R, Garrett-Mayer E. Development in infants with autism spectrum disorders: a prospective study. J Child Psychol Psychiatry. 2006;47(6):629-638

24. Ozonoff S, Iosif A, Baguio F, et al. A prospective study of the emergence of early behavioral signs of autism. J Am Acad Child Adolesc Psychiatry. 2010;49(3): 258-268

25. Yirmiya N, Gamliel I, Pilowsky T, Feldman R, Baron-Cohen S, Sigman M. The development of siblings of children with autism at 4 and 14 months: social engagement, communication, and cognition. J Child Psychol Psychiatry. 2006;47(5):511-523

26. Zwaigenbaum L, Bryson S, Rogers T, Roberts W, Brian J, Szatmari P. Behavioral manifes- tations of autism in the first year of life. Int $J$ Dev Neurosci. 2005;23(2-3):143-152

27. Fombonne E. Epidemiological trends in rates of autism. Mol Psychiatry. 2002; 7(Suppl 2):S4-S6

28. Honda H, Shimizu Y, Imai M, Nitto Y. Cumulative incidence of childhood autism: a total population study of better accuracy and precision. Dev Med Child Neurol. 2005;47(1): 10-18

29. Amor DJ, Cameron C. PGD gender selection for non-Mendelian disorders with unequal sex incidence. Hum Reprod. 2008;23(4): 729-734

30. Simonoff E. Genetic counseling in autism and pervasive developmental disorders. J Autism Dev Disord. 1998;28(5):447-456

31. Selkirk CG, Veach PM, Lian F, Schimmenti L, LeRoy BS. Parent's perceptions of autism spectrum disorder etiology and recurrence risk and effects of their perceptions on family planning: recommendations for genetic counselors. J Genet Couns. 2009;18(5): 507-519

32. Whitelaw C, Flett P, Amor DJ. Recurrence risk in autism spectrum disorder: a study of parental knowledge. J Paediatr Child Health. 2007;43(11):752-754

33. McMahon WM, Baty BJ, Botkin J. Genetic counseling and ethical issues for autism. Am J Med Genet C Semin Med Genet. 2006; 142C(1):52-57

34. Miller DT, Adam MP, Aradhya S, et al. Consensus statement: chromosomal microarray is a first-tier clinical diagnostic test for individuals with developmental disabilities or congenital anomalies. Am J Hum Genet. 2010;86(5):749-764

35. Oosterling IJ, Wensing M, Swinkels SH, et al. Advancing early detection of autism spectrum disorder by applying an integrated two-stage screening approach. J Child Psychol Psychiatry. 2010;51(3): 250-258

36. Reznick JS, Baranek GT, Reavis S, Watson LR, Crais ER. A parent-report instrument for identifying one-year-olds at risk for an eventual diagnosis of autism: the first year inventory. J Autism Dev Disord. 2007;37(9): 1691-1710

37. Robins DL, Fein D, Barton ML, Green JA. The Modified Checklist for Autism in Toddlers: an initial study investigating the early detection of autism and pervasive developmental disorders. J Autism Dev Disord. 2008;38(5):827-839

38. Wetherby AM, Brosnan-Maddox S, Peace $V$, Newton L. Validation of the Infant- 
Toddler Checklist as a broadband screener for autism spectrum disoreders from 9 to 24 months of age. Autism. 2008; 12(5) :487-511

39. Dawson G, Rogers S, Munson J, et al. Randomized, controlled trial of an intervention for toddlers with autism: the Early Start Denver Model. Pediatrics. 2010;125(1). Available at: www.pediatrics.org/cgi/ content/full/125/1/e17

40. Rogers SJ, Vismara LA. Evidence-based comprehensive treatments for early au- tism. J Clin Child Adolesc Psychol. 2008; 37(1):8-38

41. Zwaigenbaum L, Bryson S, Lord C, et al. Clinical assessment and management of toddlers with suspected ASD: insights from studies of highrisk infants. Pediatrics. 2009;123(5):1383-1391

\section{(Continued from first page)}

www.pediatrics.org/cogi/doi/10.1542/peds.2010-2825

doi:10.1542/peds.2010-2825

Accepted for publication May 5, 2011

Address correspondence to Sally Ozonoff, PhD, MIND Institute, University of California Davis Health System, 2825 50th St, Sacramento, CA 95817. E-mail: sally. ozonoff@ucdmc.ucdavis.edu

PEDIATRICS (ISSN Numbers: Print, 0031-4005; Online, 1098-4275).

Copyright (C) 2011 by the American Academy of Pediatrics

FINANCIAL DISCLOSURE: The authors have indicated they have no personal financial relationships relevant to this article to disclose.

Funded by the National Institutes of Health (NIH). 